\title{
Inhibition of PGC-1 $\alpha$ after chemotherapy-mediated insult confines multiple myeloma cell survival by affecting ROS accumulation
}

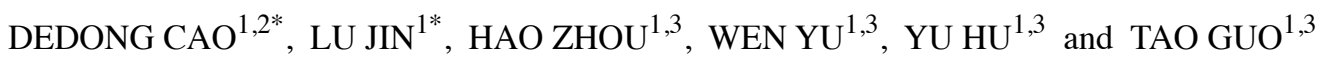 \\ ${ }^{1}$ Institute of Hematology, Union Hospital, Tongji Medical College, Huazhong University of Science and Technology; \\ ${ }^{2}$ Institute of Cancer, Renmin Hospital of Wuhan University, Wuhan University; ${ }^{3}$ Collaborative Innovation Center \\ of Hematology, Huazhong University of Science and Technology, Wuhan, Hubei 430022, P.R. China
}

Received July 30, 2014; Accepted November 7, 2014

DOI: $10.3892 /$ or.2014.3635

\begin{abstract}
Peroxisome proliferator-activated receptor- $\gamma$ coactivator-1 $\alpha$ (PGC-1 $\alpha)$ is a key regulator of reactive oxygen species (ROS). However, whether it has the same role in multiple myeloma (MM), especially after treatement with chemotherapy, remains unclear. After treating cells with bortezomib or dexamethasone, the expression of PGC-1 $\alpha$, superoxide dismutase 2 (SOD-2) and catalase (CAT) was examined by RT-PCR. PGC-1 $\alpha$ expression was also analyzed by western blotting. Small-interference RNA (siRNA) was applied to inhibit the expression of PGC-1 $\alpha$ after chemotherapy. Changes of cellular ROS and apoptosis were detected by flow cytometric analysis. Cell proliferation was assessed by MTT assay. The expression of PGC-1 $\alpha$ and SOD-2 following chemotherapy were upregulated, but accompanied by increased ROS. Following suppression of PGC-1 $\alpha$, ROS levels, as well as the pro-apoptotic effect of bortezomib were further increased. These findings suggested that $\mathrm{PGC}-1 \alpha$ regulates ROS in MM, and that inhibition of elevated PGC- $1 \alpha$ following stimulation by chemotherapy leads to a higher level of ROS by downregulating antioxidant factors, eventually enhancing the antitumor effect of bortezomib.
\end{abstract}

\section{Introduction}

Multiple myeloma (MM) is a hematological malignant disease characterized by abnormal bone marrow plasma cell proliferation (1). Despite the fact that the application of new chemotherapeutic agents has led to great progress in the treat-

Correspondence to: Dr Tao Guo, Institute of Hematology, Union Hospital, Tongji Medical College, Huazhong University of Science and Technology, 1277 Jie-fang Dadao, Wuhan, Hubei 430022, P.R. China

E-mail: guotao1968@163.com

*Contributed equally

Key words: multiple myeloma, $\mathrm{PGC}-1 \alpha$, reactive oxygen species, bortezomib, chemotherapy ment of MM, it remains incurable. The outcome or prognosis for MM patients with traditional or cytotoxic chemotherapeutic agents has not been satisfactory (2). Therefore, the identification of novel targets for MM patients to improve outcome is crucial.

Peroxisome proliferator activated receptor- $\gamma$ coactivator- $1 \alpha(\mathrm{PGC}-1 \alpha)$ is a transcriptional coactivator that regulates the genes participating in energy metabolism and mitochondrial biogenesis (3). After years of investigation regarding the onset of malignant diseases, it is recognized that PGC- $1 \alpha$ is also a master integrator of external signals such as reactive oxygen species (ROS), which is formed during cellular metabolism, but upregulated when experiencing cellular stress $(4,5)$, cold exposure (6), endurance exercise (7), and changes of cAMP (8). Increasingly, investigators have focused their attention on examining the role of PGC-1 $\alpha$ in tumors (9-13). Results of those studies reveal that PGC-1 $\alpha$ has dynamic, diverse, even paradoxical roles in tumor development by promoting cancer cell survival under cellular stress and enhancing the apoptosis of cancer cells through the coordinated regulation of Bcl-2 and Bax expression. In a previously published study, we found that PGC- $1 \alpha$ is overexpressed in MM, and PGC- $1 \alpha$ integrates angiogenesis and glucose metabolism by regulating VEGF and GLUT-4 (14). Consequently, PGC-1 $\alpha$ appears to have a pro-cancer role in MM. However, whether PGC-1 $\alpha$ has other roles in the development of $\mathrm{MM}$ remains to be elucidated.

ROS are the by-products of cell metabolism. The primary source of cellular ROS is mitochondrial. Various antioxidant systems exist for removing ROS in cells, with catalase (CAT), glutathione peroxidase 1 (GPX-1), glutathione peroxidase 4 (GPX-4), superoxide dismutase-1 (SOD-1), and superoxide dismutasis-2 (SOD-2) being the key components of antioxidant systems in cells. Cells in oxidative condition are usually considered as generating excessive ROS, and thus leading to cell or organ damage $(15,16)$. Once the generation and accumulation of ROS is out of control, it may finally lead to oxidative stress, even apoptosis $(17,18)$. Numerous studies have been performed with the aim of improving the antitumor effect of chemotherapeutic agents, and the results showed that ROS are important factors affecting the outcome for patients with tumors such as MM (19-21). Specifically, strategies 
with the aim of improving efficacy by modulating ROS have been proven to be feasible in the treatment of tumors (22). As mentioned above, PGC- $1 \alpha$ participates in the regulation of ROS, and it has been suggested that PGC-1 $\alpha$ induces several key ROS detoxifying enzymes when non-malignant cells experience oxidative stress $(4,23)$. However, whether PGC- $1 \alpha$ is involved in the regulation of ROS in MM, which is a potential target for improving the anticancer effect of chemotherapeutic agents remains to be determined.

In the present study, we cultured RPMI-8226, U266 and ARH77 MM cells in vitro, and treated these cells with bortezomib or dexamethasone. The expression of PGC- $1 \alpha$ and the key factors involved in the regulation of ROS were examined, to study whether PGC- $1 \alpha$ affected metabolism of ROS and thus influence the efficacy of chemotherapeutic agents in MM and its related underlying mechanism.

\section{Materials and methods}

Cell culture. RPMI-8226, U266 and ARH77 MM cell lines were purchased from the American Type Culture Collection (Manassas, VA, USA). Human RPMI-8226, U266 and ARH77 cells were grown in RPMI-1640 medium (Hyclone, Thermo Fisher Scientific, Waltham, MA, USA) containing $10 \%$ fetal bovine serum and $100 \mathrm{U} / \mathrm{ml}$ penicillin/streptomycin. All the cells were incubated at $37^{\circ} \mathrm{C}$ under $5 \% \mathrm{CO}_{2}$.

siRNA. The small-interference RNAs (siRNAs) for PGC-1 $\alpha$ were designed and produced by GenePharma (Shanghai, China). RPMI-8226 and ARH-77 cells were transfected with Lipofectamine $2000^{\mathrm{TM}}$ reagent (Invitrogen, Carlsabad, CA, USA) according to the manufacturer's instructions. Cells were collected $24 \mathrm{~h}$ post-transfection. The sequence information for siRNA included: GAPDH siRNA, sense control DNA and antisense control DNA (provided with the kit); siPGC-1 $\alpha, 5$ '-GCCA AACCAACAACUUUAUUU-3' (sense), and 5'-AUAAAGUUG UUGGUUUGGCUU-3' (antisense).

MTT assay. Myeloma cells were seeded in 96-well plates at a density of $3.5-5 \times 10^{4}$ cells per well. Following treatment with different doses of agents for $24 \mathrm{~h}, 10 \mu \mathrm{l}$ MTT [3-(4,5-dimethylthiazol-2-yl)-2,5-diphenyltetrazolium bromide] solution was added to each well. The plates were incubated for $4 \mathrm{~h}$ in the dark and detergent reagent was added until the purple formazan reached full dissolution. The assay was measured by Microplate Reader (Bio-Rad, La Jolla, CA, USA) and absorbance at $490 \mathrm{~nm}$ was detected. The inhibition rate was defined as: Inhibition rate $=($ OD control - OD drug - OD background $) /$ (OD control - OD background) x $100 \%$.

cDNA synthesis and quantitative PCR analysis. Total RNA was extracted from myeloma cells using TRIzol reagent (Invitrogen). cDNA was then synthesized using cDNA Reverse Transcription kit (Toyobo, Osaka, Japan). Quantitative RT-PCR was performed using the SYBR-Green PCR system on a Life ABI 7500 detection system, and results of genes of interest were normalized to $\beta$-actin. Primer sequences of genes including $\beta$-actin, $P G C$ - $1 \alpha$, catalase $(C A T)$, glutathione peroxidase $1(G P X-1)$, glutathione peroxidase $4(G P X-4)$, superoxide dismutase-1 (SOD-1), and superoxide dismutase-2 (SOD-2) were: $\beta$-actin: 5'-TTCCAGCCTTCCTTCCTGG-3' (forward), and 5'-TTGCGCTCAGGAGCAAT-3' (reverse); $P G C-1 \alpha$ : 5'-TGGTGCCACCACCATCAAAGA-3' (forward), and 5'-TCA CCAAACAGCCGCAGACTG-3' (reverse); CAT: 5'-CCAGT CGGTGTATGCCTTCT-3' (forward), and 5'-GGACGCCA CATTCTCGATAAG-3' (reverse); GPX-1: 5'-CCAGTCGGT GTATGCCTTCT-3' (forward), and 5'-GGACGCCACATT CTC GATAAG-3' (reverse); GPX-4: 5'-GCTGTGGAAGTG GAT GAA-3' (forward), and 5'-GATGAGGAACTGTGG AGAG-3' (reverse); SOD-1: 5'-ACTCATCTGTTATCCTG CTAG-3' (forward), and 5'-GCCTCATAATAAGTGCCA TAC-3' (reverse); SOD-2: 5'-TCACCGAGGAGAAGTACC-3' (forward) and 5'-TTGATATGACCACCACCATT-3' (reverse).

Reactive oxygen species. Procedures for the detection of ROS followed the manufacturer's instructions. Briefly, myeloma cells treated with different chemotherapeutic agents were incubated with $10 \mu \mathrm{M}$ DCFH-DA for $30 \mathrm{~min}$ at $37^{\circ} \mathrm{C}$. Cells were rotated gently every 5 min during incubation and then washed and resuspended in PBS. The mean fluorescence intensity of DCF was detected by FACSCalibur flow cytometer and analyzed with Cell Quest software (both from BD Biosciences, Franklin Lakes, NJ, USA).

Western blotting. Western blot analysis was performed according to the method of reference (24). Briefly, the whole cell lysate as well as SDS-PAGE, electrophoretic transfer and immunoblotting were prepared as earlier described. Protein samples were analyzed by western blotting with chemiluminescence detection (ECL; Amersham Pharmacia Biotech, Inc., Piscataway, NJ, USA). Antibodies in this study were purchased from Cell Signaling. Total protein concentration was measured according to the supplier's instructions (Thermo Fisher Scientific, Inc./Pierce, Rockford, IL, USA).

Statistical analysis. Each experiment was repeated at least three times independently. Data were presented as the mean $\pm \mathrm{SD}$ values. Differences between groups were analyzed by t-test and were considered to indicate a statistically significant difference when $\mathrm{P}<0.05$.

\section{Results}

Expression of PGC-1 $\alpha$ in RPMI-8226 and U266 cells is upregulated following treatment with chemotherapy agent. Following treatment of RPMI-8226, U266, and ARH77 cells with $10 \mathrm{ng} / \mathrm{ml}$ bortezomib or $50 \mu \mathrm{M}$ dexamethasone for $24 \mathrm{~h}$, we tested the expression of PGC-1 $\alpha$, SOD-1, SOD-2, GPX-1, GPX-4 and CAT by using RT-qPCR, to observe the changes in the levels of antioxidant factors following treatment with dexamethasone or bortezomib, and to determine which factors presented the same changing pattern as PGC- $1 \alpha$. As shown in Fig. 1, RPMI-8226 cells treated with bortezomib exhibited an elevated expression of PGC-1 $\alpha(2.18 \pm 0.13$ vs. $1.00 \pm 0.10, \mathrm{p}=0.0002$ ), when compared to cells without bortezomib treatment. The level of SOD-2 was also elevated after bortezomib treatment $(1.78 \pm 0.10$ vs. $1.00 \pm 0.10, \mathrm{p}=0.0007)$. By contrast, after treating RPMI-8226 cells with dexamethasone, the expression of PGC- $1 \alpha$ was significantly decreased as compared to that of the control $(0.23 \pm 0.01$ vs. $1.00 \pm 0.10$, 

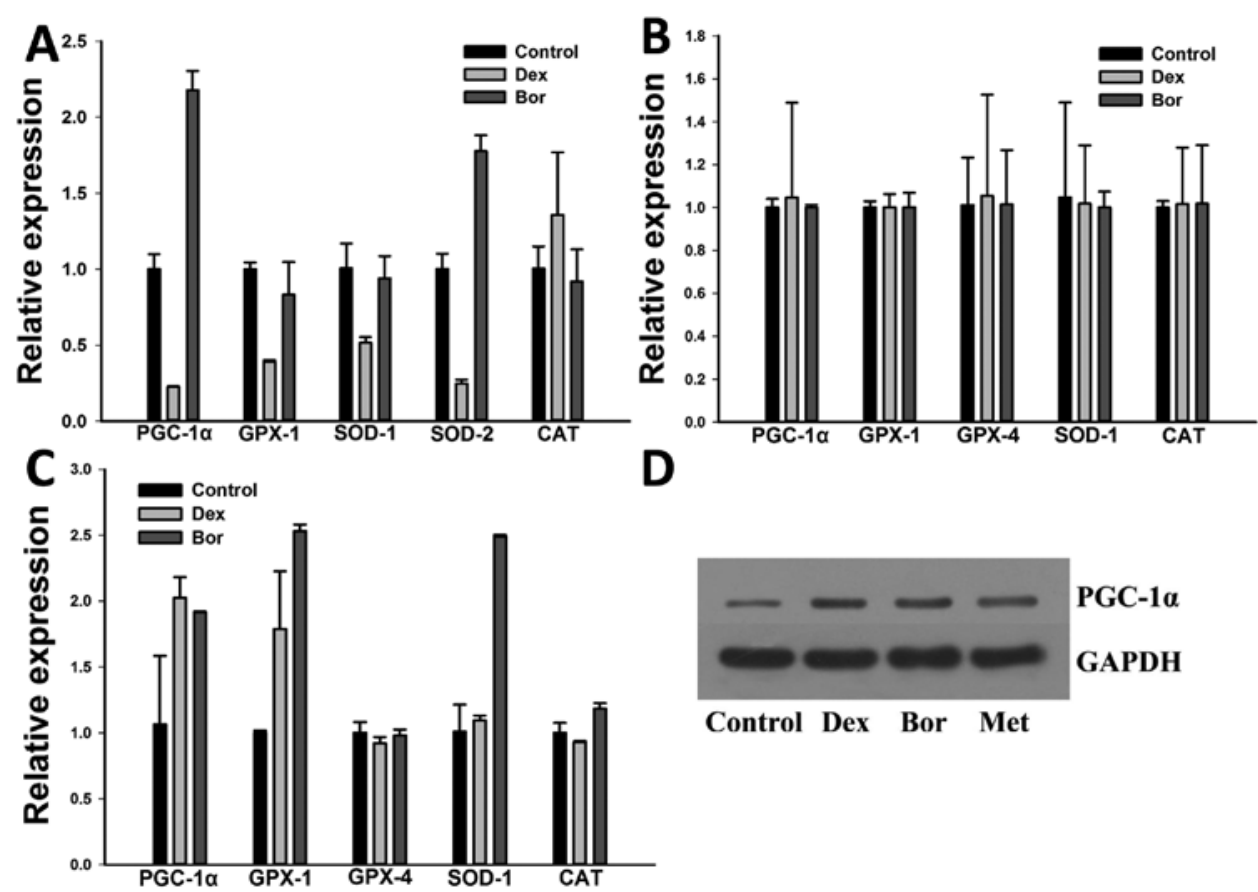

D

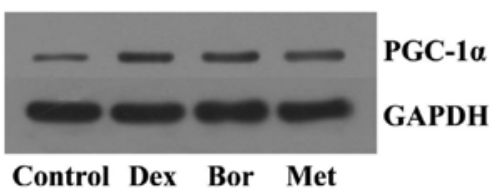

Figure 1. Expression of PGC-1 $\alpha$, SOD-1, SOD-2, GPX-1, GPX-4 and CAT in RPMI-8226, ARH77 and U266 cells after treatment. Relative expression of PGC-1 $\alpha$, SOD-1, SOD-2, GPX-1, GPX-2 and CAT mRNA in (A) RPMI-8226, (B) ARH77 and (C) U266cells after bortezomib or dexamethasone treatment. Cells were cultured in vitro and treated with $10 \mathrm{ng} / \mathrm{ml}$ bortezomib or $50 \mu \mathrm{M}$ dexamethasone for $24 \mathrm{~h}$. The expression levels of PGC-1 $\alpha$ and SOD-2 in RPMI8226 cells were significantly elevated after bortezomib treatment when compared to the control, and the levels of PGC-1 $\alpha$, GPX-1 and SOD-1 were increased after bortezomib or dexamethasone treatment. (D) Relative expression of PGC-1 $\alpha$ protein in RPMI-8226 cells after treatment of dexamethasone or bortezomib or metformin. PGC-1 $\alpha$, peroxisome proliferator activated receptor- $\gamma$ coactivator- $1 \alpha$; GPX, glutathione peroxidase; SOD, superoxide dismutase; CAT, catalase; Dex, dexamethasone; Bor, bortezomib; Met, metformin.

$\mathrm{p}=0.00018)$. Levels of other factors, such as SOD-1, SOD-2, and GPX-1, were also differentially decreased. U266 cells exhibited an upregulated expression of PGC- $1 \alpha$ after bortezomib $(1.91 \pm 0.01$ vs. $1.07 \pm 0.52, \mathrm{p}=0.048)$ or dexamethasone $(2.03 \pm 0.15$ vs. $1.07 \pm 0.52, \mathrm{p}=0.037)$ treatment. The expression levels of GPX-1, SOD-1 and CAT were also differentially increased in U266 cells treated with bortezomib. However, significant changes were not evident in the levels of PGC-1 $\alpha$, SOD-1, GPX-1, GPX-4 and CAT in ARH77 cells after treatment with dexamethasone or bortezomib. As levels of PGC-1 $\alpha$ and other antioxidant factors are highly regulated by cellular stress and therefore allow metabolic adaptation (25). Taken ogether, these results indicated that myeloma cells may protect themselves from suffering chemotherapy-induced cellular stress by enhancing the expression of one or more of the antioxidative factors.

$P G C-1 \alpha$ participates in the regulation of $S O D-2$ and $C A T$ in $M M$ cells. SOD-2 is an effective regulator of cellular ROS, and studies have reported that PGC-1 $\alpha$ is an important regulator of SOD-2 $(26,27)$. However, whether the increased expression of SOD-2 after chemotherapy is mediated by PGC- $1 \alpha$ remains to be determined. Thus, we explored the impact of PGC-1 $\alpha$ on the expression of SOD-2 as well as other key components of antioxidant systems such as GPX-1 and CAT in MM RPMI-8226 cells after chemotherapy. As shown in Fig. 2, in cells treated with siPGC- $1 \alpha$, the level of PGC-1 $\alpha$ was significantly decreased $(0.57 \pm 0.12$ vs. $1.01 \pm 0.19, p=0.027)$, and subsequently resulted in reduced expression of SOD-2 $(0.69 \pm 0.16$ vs. $1.03 \pm 0.29$, $\mathrm{p}=0.15)$, and GPX-1 (0.60 \pm 0.07 vs. $1.00 \pm 0.097, \mathrm{p}=0.0044)$ when compared to the siControl. These results suggested that PGC-1 $\alpha$ may maintain the same effect on the regulation of antioxidant systems in MM.

Suppression of PGC-1 $\alpha$ by siRNA increases the levels of ROS and enhances toxicity of bortezomib in vitro. To elucidate the role of PGC- $1 \alpha$ in the regulation of ROS, we suppressed the expression of PGC-1 $\alpha$ in RPMI-8226 and ARH77 cells with siRNA-targeting PGC-1 $\alpha$. Then, we tested the cellular ROS level by FACSCalibur flow cytometer. As shown in Fig. 3, the levels of ROS in siPGC- $1 \alpha$ cells were $\sim 1.60$-fold higher than those in the siControl cells $(1.60 \pm 0.38)$, suggesting that inhibition of the upregulated expression of PGC- $1 \alpha$ following treatment with chemotherapy may result in MM cells suffering more ROS.

A number studies have reported that the anticancer effect of bortezomib involves ROS (28-30). We tested whether elevated ROS levels enhanced the pro-apoptotic effect of bortezomib. The MTT results showed that suppression of PGC- $1 \alpha$ resulted in an enhanced inhibitory effect of bortezomib on proliferation or survival (Fig. 4).

\section{Discussion}

The influence of PGC-1 $\alpha$ on the antitumor effect of bortezomib in malignant proliferating cells, as well as how PGC-1 $\alpha$ affects outcomes of MM during treatment, remains poorly understood. In the present study, we show that the expression of PGC- $1 \alpha$ in MM cells was increased following bortezomib treatment, accompanied by upregulated levels of 

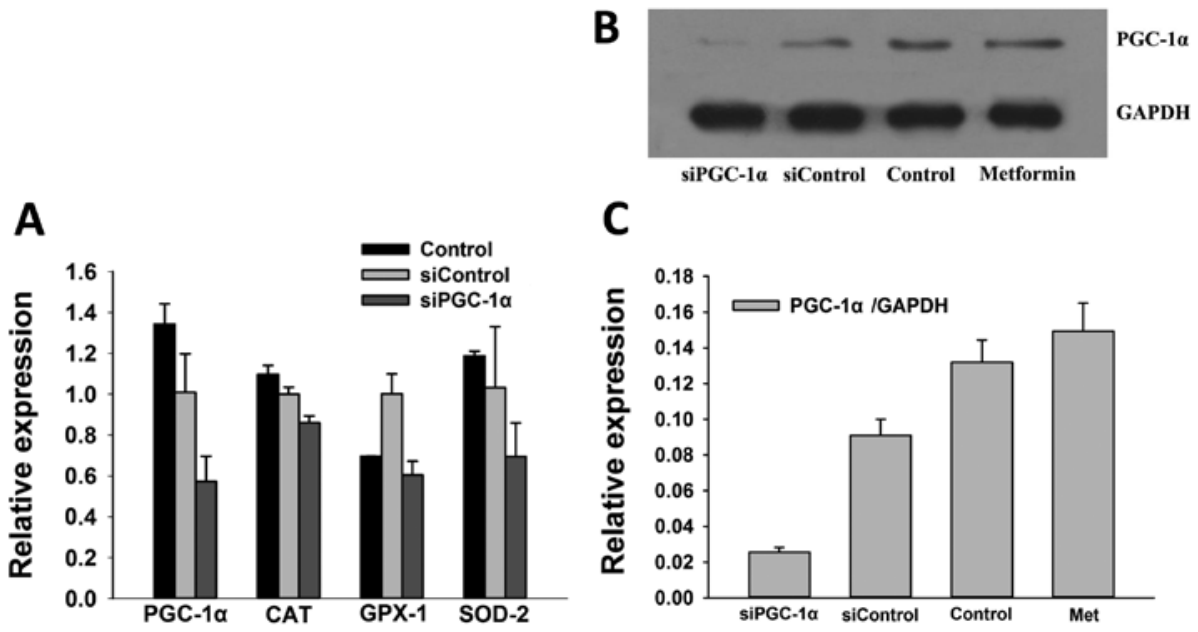

C

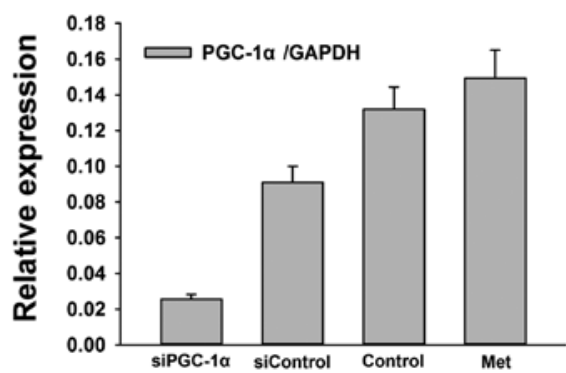

Figure 2. The expression of PGC-1 $\alpha$, CAT, GPX-1 and SOD-2 after siRNA treatment. (A-C) Suppression of PGC-1 $\alpha$ by siRNA resulted in a decreased expression of PGC-1 $\alpha$, CAT, GPX-1 and SOD-2. (A) Inhibition of PGC-1 $\alpha$ expression by siRNA in RPMI-8226 cells caused differential downregulation of PGC-1 $\alpha$, CAT, GPX-1 and SOD-2, when compared to siControl. (B and C) The expression of PGC-1 $\alpha$ in RPMI-8226 cells was inhibited by siRNA.
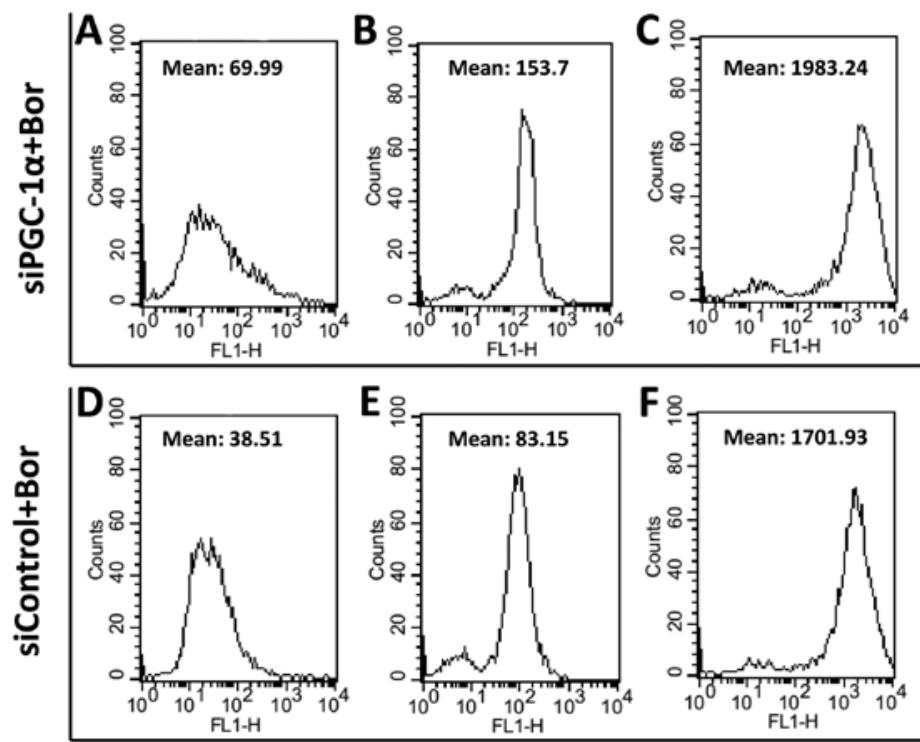

Figure 3. Changes of reactive oxygen species (ROS) in RPMI- 8226 cells after siRNA treatment. RPMI- 8226 cells treated with siControl were used as the control. The levels of ROS were tested in RPMI-8226 cells after treatment with bortezomib for $24 \mathrm{~h}$. (A-C) siPGC-1 $\alpha$ combined with bortezomib. (D-F) siControl combined with bortezomib. The levels of ROS in siPGC-1 $\alpha$ - and bortezomib-treated RPMI- 8226 cells were $\sim 1.6$-fold higher than those in the control.

A

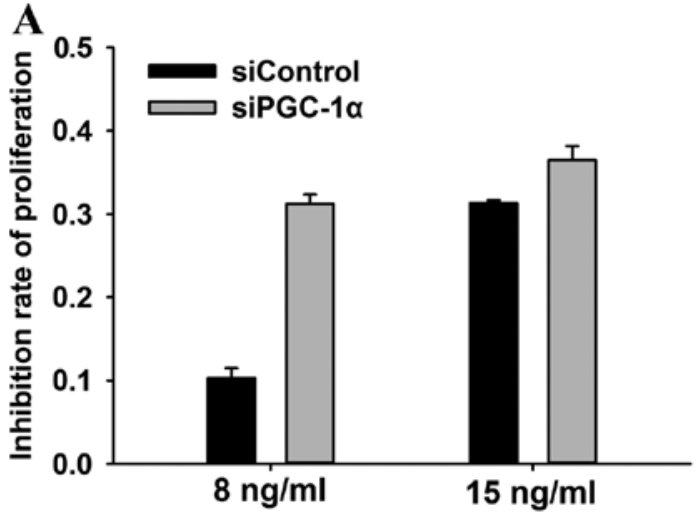

B

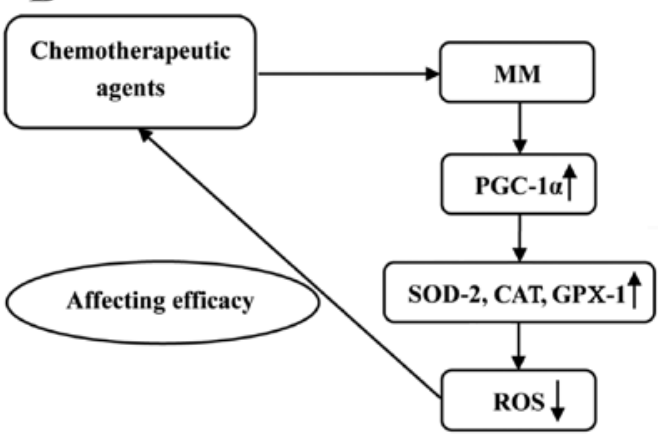

Figure 4. The antitumor effect of bortezomib is enhanced by inhibition of PGC-1 $\alpha$. After interfering the expression of PGC-1 $\alpha$ with siRNA, the inhibition rate of proliferation in cells treated with siControl was lower than that in cells treated with siPGC-1 $\alpha$, at 8 or $15 \mathrm{ng} / \mathrm{ml}$ of bortezomib. (A) Inhibition rates of proliferation. (B) The role of PGC-1 $\alpha$ in MM. MM cells exhibit upregulated PGC-1 $\alpha$ and antioxidant factors in response to treatment with chemotherapeutic agents, accompanied with decreased levels of ROS, which may be beneficial for the survival of tumor cells during chemotherapy. 
SOD-2 and CAT. Inhibition of PGC-1 $\alpha$ resulted in the downregulated expression of SOD-2 and CAT, following increased ROS and enhanced efficacy of bortezomib. The results suggest that upregulated PGC-1 $\alpha$ levels may be responsible for the impaired effect of bortezomib in the treatment of MM.

PGC-1 $\alpha$ is a transcriptional coactivator recently recognized as an important regulator of lipid metabolism, mitochondrial biogenesis, and glucose metabolism (3,31). As PGC-1 $\alpha$ has an increasingly important role in regulating the metabolism of non-malignant cells, investigators have focused their attention on the role of PGC-1 $\alpha$ in cancer $(11,32-34)$. Studies have determined that PGC-1 $\alpha$ has both pro- and anticancer functions and suggest a dynamic role of PGC-1 $\alpha$ in cancer (33). In our study, the cells treated with bortezomib showed a higher level of PGC-1 $\alpha$, a higher expression of SOD-2 and CAT was identified in these cells during chemotherapy than in those without treatment, supporting the hypothesis that PGC-1 $\alpha$ promote myeloma cell survival in conditions of chemotherapy. Furthermore, we suppressed the expression of PGC- $1 \alpha$ in myeloma cells by siRNA and these cells showed decreased expression levels of SOD-2 and CAT, accompanied by increased ROS and enhanced toxicity of the chemotherapy agents. This finding suggests that PGC-1 $\alpha$ has a pro-survival role in MM cells. We also found that genes encoding other antioxidant factors (SOD-1, GPX-4 and TRXP-1 for example) increased (data not shown) independently of PGC-1 $\alpha$. This result suggests there are pathways regulating antioxidant factors being activated in myeloma cells during chemotherapeutic stimulation.

Mitochondria are a major source of ROS in cells, and excessive generation of ROS in mitochondria is important in the development of several diseases, particularly cancer, and ageing $(18,35,36)$. PGC-1 $\alpha$ prompts mitochondrial biogenesis and enhances functions of mitochondria in muscle cells $(3,31)$, and it seems controversial that mitochondrial biogenesis induced by PGC-1 $\alpha$ may produce more ROS and lead to more effective efficacy of chemotherapy, while PGC- $1 \alpha$ suppresses ROS accumulation and results in the impaired effect of chemotherapy. This may be explained by the Warburg effect. The Warburg effect refers to an enhanced anaerobic glycolysis even in normal oxygen level conditions (37). Studies have proved that the Warburg effect is operative in MM cells $(37,38)$, and this may avoid the excessive production of ROS. Previous studies have shown that increased mitochondrial biogenesis utilizes less oxygen and produces less ROS $(39,40)$. Thus, from these aspects, the upregulation of PGC- $1 \alpha$ did not provide obstacles for the survival of MM cells.

Several studies have reported that the antitumor effect of bortezomib can be influenced by ROS (19-21,28-30,41). Pei et al used bortezomib/HDAC inhibitor regimen to treat myeloma cells, tested the changes of ROS and apoptotic factors in these cells, and found that the regimen markedly induced ROS generation and apoptosis in human MM cells (19). The study by Feng et al suggested that a bortezomib and PXD101 regimen induced cell death in MM cells via ROS-mediated DNA damage (21). Pérez-Galán et al showed that bortezomib induced mitochondrial depolarization and ROS generation in mantle-cell lymphoma (41). The above mentioned studies demonstrated that the pro-apoptotic effect of bortezomib can be enhanced by increasing ROS in tumor cells. In the present study, this was achieved by suppression of PGC-1 $\alpha$, which led to decreased levels of SOD-2 and CAT, and elevated levels of ROS.

In conclusion, our results suggest that MM cells experiencing chemotherapy may induce the expression of PGC-1 $\alpha$ and a set of genes of antioxidant factors (SOD-2, CAT) in a PGC-1 $\alpha$-dependent manner to reduce ROS accumulation. Inhibition of PGC-1 $\alpha$ is sufficient to improve the efficacy of bortezomib by increasing ROS.

\section{Acknowledgements}

This study was supported by the HuBei Provincial Natural Science Fund Subject (2013CFB096).

\section{References}

1. Rajkumar SV: Multiple myeloma: 2013 update on diagnosis, risk-stratification, and management. Am J Hematol 88: 226-235, 2013.

2. Allegra A, Penna G, Alonci A, et al: Monoclonal antibodies: potential new therapeutic treatment against multiple myeloma. Eur J Haematol 90: 441-468, 2013.

3. Wu Z, Puigserver P, Andersson U, et al: Mechanisms controlling mitochondrial biogenesis and respiration through the thermogenic coactivator PGC-1. Cell 98: 115-124, 1999.

4. St-Pierre J, Drori S, Uldry M, et al: Suppression of reactive oxygen species and neurodegeneration by the PGC-1 transcriptional coactivators. Cell 127: 397-408, 2006.

5. Austin S and St-Pierre J: PGC1 $\alpha$ and mitochondrial metabolism - emerging concepts and relevance in ageing and neurodegenerative disorders. J Cell Sci 125: 4963-4971, 2012.

6. Liang $\mathrm{H}$ and Ward WF: PGC-1alpha: a key regulator of energy metabolism. Adv Physiol Educ 30: 145-151, 2006.

7. Wrann CD, White JP, Salogiannnis J, et al: Exercise induces hippocampal BDNF through a PGC-1 $\alpha /$ FNDC5 pathway. Cell Metab 18: 649-659, 2013.

8. Puigserver $\mathrm{P}$ and Spiegelman BM: Peroxisome proliferatoractivated receptor-gamma coactivator 1 alpha (PGC-1 alpha): transcriptional coactivator and metabolic regulator. Endocr Rev 24: 78-90, 2003.

9. Kim MS, Sweeney TR, Shigenaga JK, et al: Tumor necrosis factor and interleukin 1 decrease RXRalpha,PPARalpha, PPARgamma, LXRalpha, and the coactivators SRC-1, PGC-1alpha, and PGC-1beta in liver cells. Metabolism 56: 267-279, 2007.

10. Zhang Y, Ba Y, Liu C, et al: PGC-1alpha induces apoptosis in human epithelial ovarian cancer cells through a PPARgammadependent pathway. Cell Res 17: 363-373, 2007.

11. Salem AF, Whitaker-Menezes D, Howell A, Sotgia F and Lisanti MP: Mitochondrial biogenesis in epithelial cancer cells promotes breast cancer tumor growth and confers autophagy resistance. Cell Cycle 11: 4174-4180, 2012.

12. Do MT, Kim HG, Choi JH and Jeong HG: Metformin induces microRNA-34a to downregulate the Sirt1/Pgc-1 $\alpha / \mathrm{Nrf} 2$ pathway, leading to increased susceptibility of wild-type p53 cancer cells to oxidative stress and therapeutic agents. Free Radic Biol Med 74: 21-34, 2014.

13. McGuirk S, Gravel SP, Deblois G, et al: PGC-1 $\alpha$ supports glutamine metabolism in breast cancer. Cancer Metab 1: 22, 2013.

14. Cao D, Zhou H, Zhao J, et al: PGC-1 $\alpha$ integrates glucose metabolism and angiogenesis in multiple myeloma cells by regulating VEGF and GLUT-4. Oncol Rep 31: 1205-1210, 2014.

15. Nishikawa T, Edelstein D, Du XL, et al: Normalizing mitochondrial superoxide production blocks three pathways of hyperglycaemic damage. Nature 404: 787-790, 2000.

16. Valle I, Alvarez-Barrientos A, Arza E, et al: PGC-1alpha regulates the mitochondrial antioxidant defense system in vascular endothelial cells. Cardiovasc Res 66: 562-573, 2005.

17. Edeas M: Strategies to target mitochondria and oxidative stress by antioxidants: key points and perspectives. Pharm Res 28: 2771-2779, 2011.

18. Vurusaner B, Poli G and Basaga H: Tumor suppressor genes and ROS: complex networks of interactions. Free Radic Biol Med 52: 7-18, 2012. 
19. Pei XY, Dai Y and Grant S: Synergistic induction of oxidative injury and apoptosis in human multiple myeloma cells by the proteasome inhibitor bortezomib and histone deacetylase inhibitors. Clin Cancer Res 10: 3839-3852, 2004.

20. Pei XY, Dai Y and Grant S: The proteasome inhibitor bortezomib promotes mitochondrial injury and apoptosis induced by the small molecule Bcl-2 inhibitor HA14-1 in multiple myeloma cells. Leukemia 17: 2036-2045, 2003.

21. Feng R, Oton A, Mapara MY, et al: The histone deacetylase inhibitor, PXD101, potentiates bortezomib-induced anti-multiple myeloma effect by induction of oxidative stress and DNA damage. Br J Haematol 139: 385-397, 2007.

22. Wang $\mathrm{J}$ and $\mathrm{Yi} \mathrm{J}$ : Cancer cell killing via ROS: to increase or decrease, that is the question. Cancer Biol Ther 7: 1875-1884, 2008.

23. Kong X, Wang R, Xue Y, et al: Sirtuin 3, a new target of PGC-1alpha, plays an important role in the suppression of ROS and mitochondrial biogenesis. PloS One 5: e11707, 2010.

24. Pearce EL, Walsh MC, Cejas PJ, et al: Enhancing CD8 T-cell memory by modulating fatty acid metabolism. Nature 460: 103-107, 2009.

25. Wenz T: Regulation of mitochondrial biogenesis and PGC-1 $\alpha$ under cellular stress. Mitochondrion 13: 134-142, 2013.

26. Leick L, Lyngby SS, Wojtaszewski JF and Pilegaard H: PGC-1alpha is required for training-induced prevention of ageassociated decline in mitochondrial enzymes in mouse skeletal muscle. Exp Gerontol 45: 336-342, 2010.

27. Qu A, Jiang C, Xu M, et al: PGC-1alpha attenuates neointimal formation via inhibition of vascular smooth muscle cell migration in the injured rat carotid artery. Am J Physiol Cell Physiol 297: C645-C653, 2009.

28. Hong YS, Hong SW, Kim SM, et al: Bortezomib induces G2-M arrest in human colon cancer cells through ROS-inducible phosphorylation of ATM-CHK1. Int J Oncol 41: 76-82, 2012.

29. Song IS, Jeong YJ, Jeong SH, et al: Combination treatment with 2-methoxyestradiol overcomes bortezomib resistance of multiple myeloma cells. Exp Mol Med 45: e50, 2013.

30. Nakata W, Hayakawa Y, Nakagawa $\mathrm{H}$, et al: Anti-tumor activity of the proteasome inhibitor bortezomib in gastric cancer. Int $\mathbf{J}$ Oncol 39: 1529-1536, 2011
31. Finck BN and Kelly DP: PGC-1 coactivators: inducible regulators of energy metabolism in health and disease. J Clin Invest 116: 615-622, 2006

32. Tennakoon JB, Shi Y, Han JJ, et al: Androgens regulate prostate cancer cell growth via an AMPK-PGC-1alpha-mediated metabolic switch. Oncogene: Nov 4, 2013 (Epub ahead of print). doi: 10.1038/onc.2013.463.

33. Girnun GD: The diverse role of the PPAR $\gamma$ coactivator 1 family of transcriptional coactivators in cancer. Semin Cell Dev Biol 23: 381-388, 2012.

34. Huang $\mathrm{H}$ and Manton KG: The role of oxidative damage in mitochondria during aging: a review. Front Biosci 9: 1100-1117, 2004.

35. Scarpulla RC, Vega RB and Kelly DP: Transcriptional integration of mitochondrial biogenesis. Trends Endocrinol Metab 23: 459-466, 2012.

36. Radak Z, Zhao Z, Koltai E, Ohno H and Atalay M: Oxygen consumption and usage during physical exercise: the balance between oxidative stress and ROS-dependent adaptive signaling. Antioxid Redox Signal 18: 1208-1246, 2013.

37. Sanchez WY, McGee SL, Connor T, et al: Dichloroacetate inhibits aerobic glycolysis in multiple myeloma cells and increases sensitivity to bortezomib. Br J Cancer 108: 1624$1633,2013$.

38. Fujiwara S, Kawano Y, Yuki H, et al: PDK1 inhibition is a novel therapeutic target in multiple myeloma. Br J Cancer 108: 170-178, 2013.

39. Civitarese AE, Carling S, Heilbronn LK, et al: Calorie restriction increases muscle mitochondrial biogenesis in healthy humans. PLoS Med 4: e76, 2007.

40. Lopez-Lluch G, Hunt N, Jones B, et al: Calorie restriction induces mitochondrial biogenesis and bioenergetic efficiency. Proc Natl Acad Sci USA 103: 1768-1773, 2006.

41. Pérez-Galán P, Roue G, Villamor N, Montserrat E, Campo E and Colomer D: The proteasome inhibitor bortezomib induces apoptosis in mantle-cell lymphoma through generation of ROS and Noxa activation independent of p53 status. Blood 107: 257-264, 2006 